\title{
Therapeutic applications of three-dimensional organoid models in lung cancer
}

\author{
Chang Dong Yeo ${ }^{1,2^{*}}$, Young-Pil Yun ${ }^{1,2^{*}}$, Dong Hyuck Ahn ${ }^{1,2}$, Yongki Hwang ${ }^{1,2}$, Seung Hee Yang ${ }^{1,2}$, \\ Hyobin Won ${ }^{1,2}$, Hyeong Jun Cho ${ }^{1,2}$, Chan Kwon Park ${ }^{1,2}$, Seung Joon Kim ${ }^{1,2}$, Jong Y. Park ${ }^{3}$ \\ ${ }^{1}$ Division of Pulmonology, Department of Internal Medicine, College of Medicine, The Catholic University of Korea, Seoul, Korea \\ ${ }^{2}$ Pulmonology Lab., Postech-Catholic Biomedical Engineering Institute, College of Medicine, The Catholic University of Korea, Seoul, Korea \\ ${ }^{3}$ Department of Cancer Epidemiology, Moffitt Cancer Center, Tampa, FL, USA
}

Received: February 20, 2021

Revised: March 18, 2021

Accepted: March 28, 2021

Correspondence to:

Chan Kwon Park, MD, PhD

Division of Pulmonology and Critical

Care Medicine, Department of Internal

Medicine, Yeouido St. Mary's Hospital,

College of Medicine, The Catholic

University of Korea, 10 63-ro,

Yeongdeungpo-gu, Seoul 07345, Korea

E-mail: ckpaul@catholic.ac.kr

Seung Joon Kim, MD, PhD

Division of Pulmonology, Department of Internal Medicine, Seoul St. Mary's

Hospital, College of Medicine, The

Catholic University of Korea, 222

Banpo-daero, Seocho-gu, Seoul

06591, Korea

E-mail: cmcksj@catholic.ac.kr

*These authors contributed equally.
Lung cancer, which remains a major cause of mortality worldwide, is a histologically diverse condition and demonstrates substantial phenotypic and genomic diversity among individual patients, manifesting as both intertumoral and intratumoral heterogeneity. This heterogeneity has made it difficult to develop lung cancer models. Two-dimensional (2D) cancer cell lines have been used to study genetic and molecular alterations in lung cancer. However, cancer cell lines have several disadvantages, including random genetic drift caused by long-term culture, a lack of annotated clinical data, and most importantly, the fact that only a subset of tumors shows $2 \mathrm{D}$ growth on plastic. Three-dimensional models of cancer have the potential to improve cancer research and drug development because they are more representative of cancer biology and its diverse pathophysiology. Herein, we present an integrated review of current information on preclinical lung cancer models and their limitations, including cancer cell line models, patient-derived xenografts, and lung cancer organoids, and discuss their possible therapeutic applications for drug discovery and screening to guide precision medicine in lung cancer research. Altogether, the success rate of generating lung cancer organoids must be improved, and a lung cancer organoid culture system is necessary to achieve the goal of designing an individualized therapeutic strategy for each lung cancer patient.

Keywords: Lung cancer; Organoid

\section{Introduction}

Lung cancer is a major public health problem and the most common cause of cancer death worldwide [1]. About $80 \%$ to $85 \%$ of lung cancers are non-small cell lung cancer (NSCLC). The main subtypes of NSCLC are adenocarcinoma, squamous cell carcinoma, and large cell carcinoma. The discovery of treatable oncogenic alterations led to the recommendation to include molecular testing in the standard approach [2]. This includes testing for mutations in the gene encoding epidermal growth factor receptor (EGFR), the B-Raf proto-oncogene, and serine/threonine kinase V600E, as well as searching for translocation in the genes encoding anaplastic lymphoma kinase and proto-oncogene 1 (ROS1). Targeted therapy against those driver mutations led

Copyright (C) 2021 The Organoid Society

This is an Open Access article distributed under the terms of the Creative Commons Attribution Non-Commercial License (http://creativecommons.org/licenses/bync/4.0/) which permits unrestricted non-commercial use, distribution, and reproduction in any medium, provided the original work is properly cited. 
to longer survival in patients with oncogenic driver mutations who received targeted therapies than among either patients with driver mutations who did not receive targeted therapies or patients without driver mutations [3]. However, platinumbased chemotherapy remains the cornerstone of treatment in patients who have advanced NSCLC without treatable oncogenic alterations. The response rate is approximately $25 \%$ to $30 \%$, the median survival is 8 to 12 months, and the 1-year survival rate is $30 \%$ to $40 \%$ in these patients [4]. This field is rapidly evolving, with multiple nuances deserving thorough discussion.

In recent years, the treatment and prognosis for patients with metastatic lung cancer have profoundly changed due to the introduction of immune checkpoint inhibitors [5]. Inhibition of programmed cell death 1 (PD-1) and programmed death ligand 1 (PD-L1) interactions can lead to restored T-cell function and antitumor activity [6]. The PD-L1 tumor proportion score is now routinely used to predict whether patients will benefit from anti-PD-1 agents and select therapy in advanced NSCLC. In addition, the tumor mutation burden has also been proposed as a potential predictive biomarker of benefit from anti-PD-1 therapy [7]. Therefore, understanding genetic alterations and the tumor immune microenvironment associated with therapeutic screening is important.

NSCLC is histologically diverse and demonstrates substantial phenotypic and genomic diversity among individual patients, manifesting as both intertumoral and intratumoral heterogeneity; this heterogeneity makes it difficult to create animal models [8]. Each tumor harbors a different mutational pattern and even tumors with the same histological appearance exhibit molecular diversity [9]. Tumor heterogeneity results in significant differences in the tumor growth rate, invasion ability, drug sensitivity, and prognosis [10]. However, a major challenge in studying NSCLC is the low quantity of cells that can be isolated from lung tissue biopsies [11]. Patient-derived lung cancer models continue to be developed to gain a better understanding of molecular pathogenesis, to identify novel therapeutic targets that may also serve as promising biomarkers, and to test novel therapeutic agents, thereby allowing personalized anti-cancer therapy in clinical settings $[12,13]$. As the establishment of a high-fidelity preclinical cancer model is urgently needed [13], cancer cell lines and patient-derived xenograft (PDX) models have been used to study genetic and molecular alteration. However, these models of human lung cancer have many limitations [14].

The term "organoid," which means "resembling an organ," was used as early as 1946, and organoids now refer to threedimensional (3D) structures composed of multiple cell types of their in vivo counterparts, similar to primary tissue and specific to the parent organ $[14,15]$. Although major advances were outlined in recent reports describing protocols for the development of lung cancer organoids (LCOs) [16-18], the establishment of pure LCOs is challenging [19]. Herein, we describe current information on preclinical lung cancer models and their limitations, including cancer cell line models, PDXs, and LCOs, and discuss their possible therapeutic applications for drug discovery and screening to guide precision medicine in lung cancer research.

\section{Preclinical models}

\section{Cancer cell line models}

Over the last decades, tremendous efforts have been made to develop preclinical models of NSCLC, including 2-dimensional (2D) cell lines and air-liquid interface cultures [20]. Cancer cell lines, which are characterized by low cost and ease of use, have been broadly employed for high-throughput screening of drug candidates and cancer biomarkers [13]. The US National Cancer Institute (NCI) and the Human Cancer Center lines are the 2 largest series of lung cancer cell lines that have been established, containing more than 200 lung cancer cell lines, of which perhaps 150 are well characterized [20]. Cultured tumor cells accurately represent tumor cells in vivo without the complex in vivo environment and are basically populations of pure tumor cells without admixed stromal or inflammatory cells [21]. The lack of stromal and inflammatory cells is practical for large-scale pharmacogenomic studies [12]. These projects include genomics, copy number variation analyses, transcriptomics, and screening for drug response in more than 100 lung cancer cell lines, as well as studies investigating associations between predictive biomarkers and drug sensitivity [22-25]. For example, EGFR tyrosine kinase inhibitors (EGFR-TKIs) have been developed to target activating EGFR mutations based on an in vitro model of mutation specificity created by calculating the ratio of IC50 values between mutated and wild-type EGFR [26]. Based on these pharmacogenomic and drug sensitivity models, we have multiple EGFR-TKI options to treat patients with lung cancer harboring activating EGFR mutations [27]. Moreover, cancer cell line models are also relatively easy to work with for genetic manipulation. CRISPRCas9 is a versatile genomic editing technology used to study the functions of genetic elements that accelerates the study of multigenic processes, such as the role of mutation combinations in tumor evolution [28]. Cas9 has been broadly applied in a variety of cell line- and embryo-based experiments [29,30]; however, the in vivo applications of Cas9 in somatic tissue remain more challenging due to a combination of factors, such as its large transgene size [28]. In addition, cell lines enable clonal selection and expansion to 
validate and select for positive knock-out cells [12].

Although cell lines are commonly used in preclinical models, they have important limitations that should be considered [31]. First, contamination of long-term cultured cells represents a major problem [20]. Second, genetic and mRNA changes have been reported in cell lines [32]. Several studies showed poorly concordant drug sensitivity results in the same cell lines with different experimental protocols $[33,34]$. Third, cancer cells no longer retain the tumor heterogeneity present in the primary cancer [31]. Cell lines are likely to represent a subpopulation of the original tumor and are largely homogeneous due to the selective survival pressures present in culture conditions [35]. Fourth, cell lines do not contain the relevant components of the tumor microenvironment [31]. The lack of interaction with stromal, immune, and inflammatory cells limits translational cancer cell line-based studies, especially immunologic research [20]. In recent years, patient-derived cancer cell culture models from tumor biopsies were established, and their immunofluorescencebased functional assays are promising in response to targeted therapy [36]. Further studies elucidating the concordance of drug responses between patients and the respective cell lines are needed.

\section{Patient-derived tumor xenograft models}

PDXs are models involving the implantation of patient-derived tumor tissue into immunodeficient mice [37]. Compared with conventional models, PDXs are characterized by the preservation of tumor heterogeneity and the tumor microenvironment (including stroma/vasculature), which are expected to enable a high ability to predict therapeutic efficacy $[37,38]$. Based on recent advances, the US NCI announced plans to switch its anticancer drug screening system to PDX-based models [39]. The engraftment rate varies greatly depending on the type of tumor. High graft survival rates ( $80 \%$ or higher) have been reported for melanoma and colorectal cancer, whereas the rate is as low as approximately $30 \%$ for breast cancer $[40,41]$.

In NSCLC, the engraftment rates range from $25 \%$ to $60 \%$, depending on 3 essential elements of PDX models: (1) the tumor properties; (2) the recipient mice; and (3) the recipient site [42-44]. Surgically resected tumors are usually established in PDX models; however, PDXs can also be created using biopsy specimens or circulating tumor cells collected from the blood, since advanced lung cancer patients seldom undergo surgical resection $[42,45]$. Moreover, the probability of the successful engraftment of PDX lesions is higher for tumors from metastatic foci or with greater malignancy potential $[46,47]$. To improve the efficacy engraftment rates, severe combined immune deficiency (SCID) mice were crossbred with non-obese diabetic (NOD) mice, yielding NOD/SCID mice with composite immunodeficiency $[48,49]$. However, NOD/SCID mice have a short lifespan and a high incidence of thymoma, and lack mature T-cell graft survival [50]. In 2002, NOD/SCID gamma (NSG) mice were developed from a NOD/SCID background with additional interleukin-2 receptor gamma chain impairment [51]. NGS mice are considered to be the best immunodeficient animal for human graft transplantation [52]. Regarding the recipient site, heterotopic implantation (e.g., subcutaneous grafting) provides the advantages of a simple procedure and accurate tumor size measurements [53]. However, orthotopic implantation provides a native tumor and metastasis environment, although it is technically challenging [54].

Although PDX models are considered promising tools for individualized cancer therapy, drug development and coclinical trials [38], several important limitations of PDX models should be considered. First, the tumor microenvironment is virtually non-existent due to the lack of stromal cells and degradation of tissue architecture [38]. The original human stromal and immune cells are replaced by mouse stromal cells after serial passages, thereby losing the contribution of human stromal cells to the original tumor biology. Immunotherapy has recently revolutionized cancer treatment; however, PDXs generated in non-humanized immunodeficient mice cannot be efficiently used to study immunotherapy. Humanized mice have been developed by intravenous injections of $\mathrm{CD} 34^{+}$cells isolated from the blood of patients into mice to reconstruct a functional system in murine models that would mimic that of patients $[55,56]$. Second, largescale experiments are difficult to perform in PDXs; in addition, genetic drift may occur after numerous passages. It is common practice to limit PDX experiments to fewer than 10 passages [57]. Third, less aggressive tumors exhibit decreased implantation rates and more aggressive tumors exhibit increased formation rates. Therefore, improvement of the implantation rate is urgently required. Fourth, substantial resources (e.g., money, time, and labor) are needed to create PDX models [37]. For example, typically, at least 3 months are required to develop PDXs that may be used for preclinical studies, and not all patients may be able to afford the high costs of PDX models, especially when using humanized mice [12].

\section{Three-dimensional lung organoids}

\section{General concepts of organoids}

Organoids are defined as 3D structures derived from organspecific stem cells that self-organize through cell sorting and spatially restricted lineage commitment in a manner reminiscent of the native organ with some degree of organ functionality $[58,59]$. 
Organoid cultures can be established from embryonic stem cells (ESCs), induced pluripotent stem cells (iPSCs), and adult stem cells (ASCs) [60]. ASC-derived organoids have been used to study models of infectious, hereditary, and oncological diseases that recapitulate the essential features of in vivo disease [60]. Although organoids were first successfully derived from the mouse small intestine using single Lgr5 ${ }^{+}$stem cells [61], organoids have been cultured from multiple endoderm-derived organs, including the human colon, prostate, and intestine [62-64]. Organoid growth requires the initiating stem cell population to self-renew, increase the organoid size, and differentiate [65].

Attempts have been made to develop artificial counterparts of in vivo organs from their tissues or cells, and organoid technology as a technological field emerged in tissue engineering [66]. Schwank et al. [67] reported functional repair of the cystic fibrosis transmembrane conductor receptor by CRISPR/Cas 9 in cultured intestinal stem cell organoids from cystic fibrosis patients. These organoids provide a powerful platform for elucidating disease development mechanisms, modeling diseases, and screening drug candidates for genetic, infectious, and malignant diseases [68]. Organoid cultures will be facilitated by the application of $3 \mathrm{D}$ printing as a new and potentially promising technology [69]. For example, organoids have been used as a promising platform to research how coronavirus disease 2019 affects humans and causes damage and to identify possible drug targets [70]. In the field of regenerative medicine, there is still a long way to go for the transplantation of organoids as therapy [71,72]. Below, we discuss the present limitations and potential future applications of organoids for research on malignancies, especially lung cancer.

\section{Lung organoids}

The lung is a complex organ comprising multiple cell types that perform a variety of vital processes, including immune defense and gas exchange [58]. The lung is comprised of 2 main compartments (airways and the alveolar space), which contain distinct stem cell populations: basal and club cells in the airway and alveolar epithelial type 2 (AT2) cells in the alveolar space [73]. Organoids providing a promising platform to investigate the function of lung epithelial and progenitor cells could be derived from basal cells, club cells, variant club cells, bronchoalveolar stem cells, and AT2 cells [14]. Human pluripotent stem cells (hPSCs), including ESCs and iPSCs, have been recently adopted for research [74]. A number of pulmonary diseases, including chronic obstructive pulmonary disease, asthma, pulmonary fibrosis, viral infectious diseases, and lung cancer, have been proposed to be associated with improper epithelial regeneration [14,75]. Epithelial damage and impaired regeneration, which result from stem cell exhaustion, contribute to recurrent pulmonary infection and persistence of inflammation, since cells that are injured vary in different lung diseases $[76,77]$. Although culture models from animal lungs have been developed [78], culture from primary human cells can be hampered by logistical challenges.

The first self-organizing 3D structure of adult human airway epithelial cells cultured on collagen was described in 1993 [79]. Airway basal cells gave rise to tracheo/bronchospheres in a 3D air-liquid interface and produced branching structures with multipotent potential $[80,81]$. The first attempt to generate hPSCderived organoids was reported in 2015, and showed that hPSCs differentiated into multi-lineage organoids, containing basal, ciliated, and club cells $[82,83]$. Lung bud organoids (LBOs), which were later induced from hPSCs, recapitulate many aspects of lung development, allowing branching morphogenesis and initial alveologenesis [84]. LBOs consist predominantly of AT2 cells that actively take up and secrete surfactant, reflecting an important function of AT2 cells. Tan et al. [85] combined human adult primary bronchial epithelial cells, lung fibroblasts, and lung microvascular epithelial cells in supportive 3D culture conditions to generate airway organoids. Mixed cell populations underwent rapid condensation to self-organize into discrete epithelial and endothelial structures that were stable for up to 4 weeks of culture. In a recent study, long-term expanding human airway organoids from bronchoalveolar resections or lavage material were established, which provided versatile models for the study of hereditary, malignant, and infectious diseases [17].

Although lung organoids currently represent the closest model to the human pulmonary system, several limitations should be considered. First of all, the absence of the immune system, circulatory system, and naïve extracellular matrix is a limitation. Current organoid matrix materials have lot-to-lot variability and spatial heterogeneity [86]. Combined with CRISPR/Cas9 technology, lung organoids can be used to model genetic diseases and test drug treatment. Most drug screening platforms require the use of the same starting material; however, organoids are selforganized tissues, and therefore are usually not uniform in size [74]. A number of outstanding challenges currently need to be addressed; however, the use of organoids combined with the novel techniques of live imaging, genetic engineering and biomaterials will hugely advance the pulmonary field.

\section{LCOs and translational research in the clinic}

\section{LCOs}

Recent advances using 3D organoid cultures derived from patient 
cells have opened the possibility to employ LCOs as tools for personalized medicine $[16-19,87,88]$. However, the success rates of long-term culture establishment vary substantially, and detailed descriptions of the number of passages achieved and split ratios used are typically not reported (Table 1) [16-19,87,88]. A major challenge when culturing cancer samples from primary intrapulmonary tumors is potential overgrowth by normal epithelial cells, limiting the overall establishment rate of pure LCOs to $17 \%$ [19]. One method to increase establishment is to treat organoid cultures with the MDM2 inhibitor nutlin-3a to selectively grow out p 53 mutant cells, resulting in pure tumor cells harboring TP53 mutations; this method is useful due to the large amount of contamination by nontumor cells [17]. Alternatively, the use of a suboptimal medium for LCO culture did not support normal cell growth, and use of feeder-free LCO lines could avoid fibroblast contamination, resulting in a 70\% success rate [87]. In addition, an understanding of biases in fibroblast outgrowth may help formulate culture media that are more permissive [89]. Intratumoral heterogeneity should be considered because the populations of cancer cells in different parts of the same tumor may exhibit different drug sensitivity; therefore, multiple sampling may be necessary [14].

LCOs derived from primary tumors should be routinely evaluated for tumor purity using genetic methods or histomorphology combined with p63 and CK5/6 staining [19]. In mixed cultures, a small subpopulation of normal airway organoids eventually dominates the culture. Repeated evaluation of tumor purity is important for mixed tumor/normal cultures. Histomorphology combined with p63 staining can also make it easier to distinguish subtype markers for lung cancer such as
TTF-1, cytokeratin 5, and synaptophysin, thereby providing a reliable classification $[16,87]$. LCOs further maintain defined genetic characteristics, including copy number profiling, singlenucleotide polymorphism genotyping, and variant allele frequency distribution [19]. Next-generation sequencing of LCOs and tumors has demonstrated the presence of matching somatic mutations, such as EGFR, KRAS, and TP53.

Recent studies substantially overcame the limitations of LCO models, which are the lack of the complexity of the immune system and vascularization, key cell types, and high-throughput workflows. LCOs may be maintained from tumor biopsies or surgical resection in both short- and long-term (more than 10 passages) culture and show strong correlations with the parental tumor in terms of gene expression $[17,88]$. Studies have recently described protocols to establish organoids from various epithelial tissues and cancers, as well as protocols to test drug sensitivity in patient-derived organoids [90]. Advances in procedure to isolate circulating tumor cells or cells in aspirated pleural effusion have made it possible to establish organoids, which help to acquire relevant genetic and epigenetic information about tumors in real time, as well as to screen and test promising drugs $[11,91,92]$. Kim et al. [87] established a living biobank of 80 LCOs derived from major lung cancer subtypes, which predict patientspecific drug response. Last, LCO modeling of tumor immune microenvironment models with endogenous immune stroma could enable immuno-oncology investigations [16].

\section{Drug screening applications}

In the past decades, many anti-cancer drugs developed by screening traditional $2 \mathrm{D}$ cell cultures as preclinical disease model

Table 1. Summary of lung cancer organoids models: published reports

\begin{tabular}{|c|c|c|c|c|}
\hline Year & Study & Success rate $(\%)$ & Source & Key feature \\
\hline \multirow[t]{2}{*}{2018} & Dijkstra et al. [18] & $6 / 6(100)$ & Resection (4/4) & Co-culture of peripheral blood lymphocytes and tumor organoid \\
\hline & & & Biopsy (2/2) & \\
\hline \multirow[t]{2}{*}{2019} & Sachs et al. [17] & $5 / 18(27.8)$ & Biopsy & Use of nutlin-3a \\
\hline & & & & $>10$ passages \\
\hline \multirow[t]{2}{*}{2019} & Kim et al. [87] & $39 / 56(69.6)$ & Resection & Suboptimal media \\
\hline & & & & Feeder-free cell lines \\
\hline \multirow[t]{2}{*}{2020} & Dijkstra et al. [19] & 10/58 (17.2) & Resection (5/28) & Overgrowth by normal airway organoid (14/58) \\
\hline & & & Biopsy $(4 / 30)$ & $\begin{array}{l}\text { Success rate: extrapulmonary lesion }(6 / 27)>\text { intrapulmonary } \\
\text { lesion }(3 / 31)\end{array}$ \\
\hline
\end{tabular}


system have proven to be ineffective in clinical studies [93]. As patient-derived lung cancer cultures enable personalized patient care [36], organoids derived from lung cancer patients can be used for further high-throughput drug screening [94]. Tumor organoid technology has the potential to predict patient response; therefore, research groups have performed drug screening on patient-derived organoids $[17,87,90,95]$. In vitro high-throughput assays using patient-derived tumor organoids were suitable for evaluating molecular-targeted drugs under conditions that better reflect pathologic conditions [96] Recently, living biobank-based genomic alterations of the original tumors have been established; therefore, a biobanking system of tumor organoids provides promising opportunities for patient-specific drug trials [87]. Wang et al. [97] showed promising antitumor activity with pyrotinib using a patient-derived organoid model from HER2-mutant lung cancer, and they validated those preclinical findings in patients enrolled in a phase II clinical trial. Based on recent advances in microfluidic-based culture platforms that can load, expand, and identify drug responses under physiologically relevant conditions, organoid drug screening assays provide important information to guide therapeutic approaches at the preclinical level [98]. These platforms have further been used in attempts to isolate and expand lung-circulating tumor cells and patient immune cells from liquid biopsies [99]. However, there has been no comparative study of organoid marker expression or drug responses in different media formulations. In addition, robust drug screening data would be highly promising for personalized medicine.

\section{Research in immuno-therapy}

Immune checkpoint inhibitors, such as anti-PD-1/PD-L1, have transformed the treatment landscape for lung cancer. However, preclinical models that incorporate both endogenous $\mathrm{T}$ cells and tumor cells are scarce. In addition, the expansion of tumorinfiltrating lymphocytes has been especially challenging for epithelial cancers [100]. While defined media led to the loss of stromal cell fraction during LCO establishment, the approach used to establish tumor-stroma organoids is to recombine stromal and parenchymal tumor fractions after culturing them separately. In 2018, Dijkstra et al. [18] showed that co-culture of autologous tumor organoids and peripheral blood lymphocytes provided a means by which to assess the sensitivity of tumor cells to T-cell mediated attack at the level of the individual patient. The procedures are as follows: (1) organoids are isolated from Geltrex (Thermo Fisher Scientific, Waltham, MA, USA) 2 days before co-culture and stimulated with interferon-gamma 1 day before co-culture; (2) on the day of co-culture, organoids are dissociated to single cells and plated together with peripheral blood mononuclear cells (PBMCs) on an anti-CD28-coated plate, in the presence of interleukin-2 and anti-PD-1; (3) after 1 week of co-culture, PBMCs are restimulated with tumor cells; and (4) after 2 weeks of co-culture with the autologous tumor organoid, T-cell reactivity is assessed by evaluating CD107a and interferongamma expression or CD137 expression in the presence or absence of tumor organoids. In addition, a tumor organoid killing assay can be assessed by live-cell imaging or flow cytometry-based quantification of live tumor cells [100]. However, lung cancer is considered to comprise hypermutated tumors, whereas organoids are often derived from small biopsies, which represent only a small part of the tumor tissues and therefore might underestimate the complexity of the entire tumor tissue [101]. Furthermore, LCOs are not exposed to the external pressures that occur in situ, such as hypoxia or immune selection, which can influence the outgrowth of tumor clones, leading a situation in which a dominant clone in vitro is not as dominant in situ and vice versa [101]. Nevertheless, in the near future, it will be possible to model the tumor immune microenvironment using a patient-derived organoid approach that preserves the original tumor $\mathrm{T}$-cell receptor spectrum and successfully models immune checkpoint inhibitors for biomarker identification, drug screening, and modeling of therapy resistance [102].

The potential use of LCOs for precision medicine is limited by their low success rate and frequent overgrowth by normal airway organoids. For all these therapeutic applications to be established, LCO success rates must be enhanced.

\section{Conclusion and further perspectives}

The use of organoids is expanding in the field of lung cancer, and progress has been made in understanding the relationship between cancer biology and genetics. Although the use of recently emerged LCO models remains in its infancy, these models provide drug screening and applications for T-cell based immunotherapy at the level of the individual patient. With a better understanding of immuno-oncology and advanced translational research, LCOs are expected to improve the concordance between drug response and actual clinical outcomes in the future. In conclusion, our review demonstrates that the success rate of generating LCOs must be improved, and a LCO platform is necessary to achieve the goal of designing a therapeutic strategy for each lung cancer patient.

\section{Notes}

\section{Conflict of interest}

Seung Joon Kim has been the editor-in-chief of Organoid since 
January 2021. No other potential conflict of interest relevant to this article was reported.

\section{Funding}

This work was supported by the Bio \& Medical Technology Development Program of the National Research Foundation of Korea (NRF) funded by the Korea government (MSIT) (No. 2019M3A9H2032425), by the NRF grant funded by the Korea government (MSIT) (No. 2020R1A2C2015140), by the Industrial Strategic Technology Development Program (20009773, Commercialization of 3D Multifunction Tissue Mimetics Based Drug Evaluation Platform) funded by the Ministry of Trade, Industry \& Energy (MOTIE, Korea) and by a grant of the Korea Health Technology R\&D Project through the Korea Health Industry Development Institute (KHIDI), funded by the Ministry of Health \& Welfare, Republic of Korea (grant number: HI21C0651).

\section{Author contributions}

Conceptualization: CDY, YPY, CKP, SJK, Methodology: all authors, Data curation: CDY, YPY, CKP, SJK, Investigation: all authors, Formal analysis: CDY, YPY, Project administration: CKP, SJK, Resources: CDY, YPY, CKP, SJK, Validation: CDY, YPY, CKP, SJK, Writing-original draft: CDY, YPY, Writing-review \& editing: all authors.

\section{ORCID}

Chang Dong Yeo, https://orcid.org/0000-0002-4103-7921

Young-Pil Yun, https://orcid.org/0000-0003-4268-1417

Dong Hyuck Ahn, https://orcid.org/0000-0003-4225-9806

Yongki Hwang, https://orcid.org/0000-0003-3387-724X

Seung Hee Yang, https://orcid.org/0000-0003-3031-4281

Hyobin Won, https://orcid.org/0000-0002-6704-5823

Hyeong Jun Cho, https://orcid.org/0000-0001-6347-245X

Chan Kwon Park, https://orcid.org/0000-0002-4107-444X

Seung Joon Kim, https://orcid.org/0000-0003-4836-8958

Jong Y. Park, https://orcid.org/0000-0002-6384-6447

\section{References}

1. Edwards BK, Noone AM, Mariotto AB, Simard EP, Boscoe FP, Henley SJ, et al. Annual report to the nation on the status of cancer, 1975-2010, featuring prevalence of comorbidity and impact on survival among persons with lung, colorectal, breast, or prostate cancer. Cancer 2014;120:1290-314.

2. Reck M, Rabe KF. Precision diagnosis and treatment for advanced non-small-cell lung cancer. N Engl J Med 2017;
377:849-61.

3. Kris MG, Johnson BE, Berry LD, Kwiatkowski DJ, Iafrate AJ, Wistuba II, et al. Using multiplexed assays of oncogenic drivers in lung cancers to select targeted drugs. JAMA 2014; 311:1998-2006.

4. Novello S, Barlesi F, Califano R, Cufer T, Ekman S, Levra MG, et al. Metastatic non-small-cell lung cancer: ESMO Clinical Practice Guidelines for diagnosis, treatment and follow-up. Ann Oncol 2016;27(suppl 5):v1-27.

5. Awad MM, Gadgeel SM, Borghaei H, Patnaik A, Yang JC, Powell SF, et al. Long-term overall survival from KEYNOTE-021 cohort G: pemetrexed and carboplatin with or without pembrolizumab as first-line therapy for advanced nonsquamous NSCLC. J Thorac Oncol 2021;16:162-8.

6. Boussiotis VA. Molecular and biochemical aspects of the PD-1 checkpoint pathway. N Engl J Med 2016;375:1767-78.

7. Li JJ, Karim K, Sung M, Le LW, Lau SC, Sacher A, et al. Tobacco exposure and immunotherapy response in $\mathrm{PD}-\mathrm{L} 1$ positive lung cancer patients. Lung Cancer 2020;150:159-63.

8. Chen Z, Fillmore CM, Hammerman PS, Kim CF, Wong KK. Non-small-cell lung cancers: a heterogeneous set of diseases. Nat Rev Cancer 2014; 14:535-46.

9. Hirsch FR, Scagliotti GV, Mulshine JL, Kwon R, Curran WJ Jr, $\mathrm{Wu} \mathrm{YL}$, et al. Lung cancer: current therapies and new targeted treatments. Lancet 2017;389:299-311.

10. McGranahan N, Swanton C. Clonal heterogeneity and tumor evolution: past, present, and the future. Cell 2017;168:613-28.

11. Mazzocchi A, Devarasetty M, Herberg S, Petty WJ, Marini F, Miller L, et al. Pleural effusion aspirate for use in 3D lung cancer modeling and chemotherapy screening. ACS Biomater Sci Eng 2019;5:1937-43.

12. Huo KG, D’Arcangelo E, Tsao MS. Patient-derived cell line, xenograft and organoid models in lung cancer therapy. Transl Lung Cancer Res 2020;9:2214-32.

13. Fan H, Demirci U, Chen P. Emerging organoid models: leaping forward in cancer research. J Hematol Oncol 2019;12:142.

14. Wang J, Li X, Chen H. Organoid models in lung regeneration and cancer. Cancer Lett 2020;475:129-35.

15. Dutta D, Heo I, Clevers H. Disease modeling in stem cell-derived 3D organoid systems. Trends Mol Med 2017;23:393410.

16. Neal JT, Li X, Zhu J, Giangarra V, Grzeskowiak CL, Ju J, et al. Organoid modeling of the tumor immune microenvironment. Cell 2018;175:1972-88.

17. Sachs N, Papaspyropoulos A, Zomer-van Ommen DD, Heo I, Böttinger L, Klay D, et al. Long-term expanding human airway organoids for disease modeling. EMBO J 2019;38:e100300. 
18. Dijkstra KK, Cattaneo CM, Weeber F, Chalabi M, van de Haar J, Fanchi LF, et al. Generation of tumor-reactive T cells by co-culture of peripheral blood lymphocytes and tumor organoids. Cell 2018;174:1586-98.

19. Dijkstra KK, Monkhorst K, Schipper LJ, Hartemink KJ, Smit EF, Kaing S, et al. Challenges in establishing pure lung cancer organoids limit their utility for personalized medicine. Cell Rep 2020;31:107588.

20. Gazdar AF, Girard L, Lockwood WW, Lam WL, Minna JD. Lung cancer cell lines as tools for biomedical discovery and research. J Natl Cancer Inst 2010;102:1310-21.

21. Sato G. Tissue culture: the unrealized potential. Cytotechnology 2008;57:111-4.

22. Barretina J, Caponigro G, Stransky N, Venkatesan K, Margolin AA, Kim S, et al. The cancer cell line encyclopedia enables predictive modelling of anticancer drug sensitivity. Nature 2012;483:603-7.

23. Garnett MJ, Edelman EJ, Heidorn SJ, Greenman CD, Dastur A, Lau KW, et al. Systematic identification of genomic markers of drug sensitivity in cancer cells. Nature 2012;483:570-5.

24. Basu A, Bodycombe NE, Cheah JH, Price EV, Liu K, Schaefer GI, et al. An interactive resource to identify cancer genetic and lineage dependencies targeted by small molecules. Cell 2013;154:1151-61.

25. Haverty PM, Lin E, Tan J, Yu Y, Lam B, Lianoglou S, et al. Reproducible pharmacogenomic profiling of cancer cell line panels. Nature 2016;533:333-7.

26. Hirano T, Yasuda H, Tani T, Hamamoto J, Oashi A, Ishioka $\mathrm{K}$, et al. In vitro modeling to determine mutation specificity of EGFR tyrosine kinase inhibitors against clinically relevant EGFR mutants in non-small-cell lung cancer. Oncotarget 2015;6:38789-803.

27. Ramalingam SS, Vansteenkiste J, Planchard D, Cho BC, Gray JE, Ohe Y, et al. Overall survival with osimertinib in untreated, EGFR-mutated advanced NSCLC. N Engl J Med 2020;382:41-50.

28. Platt RJ, Chen S, Zhou Y, Yim MJ, Swiech L, Kempton HR, et al. CRISPR-Cas9 knockin mice for genome editing and cancer modeling. Cell 2014;159:440-55.

29. Cong L, Ran FA, Cox D, Lin S, Barretto R, Habib N, et al. Multiplex genome engineering using CRISPR/Cas systems. Science 2013;339:819-23.

30. Mali P, Yang L, Esvelt KM, Aach J, Guell M, DiCarlo JE, et al. RNA-guided human genome engineering via Cas9. Science 2013;339:823-6.

31. Wilding JL, Bodmer WF. Cancer cell lines for drug discovery and development. Cancer Res 2014;74:2377-84.
32. Daniel VC, Marchionni L, Hierman JS, Rhodes JT, Devereux WL, Rudin CM, et al. A primary xenograft model of small-cell lung cancer reveals irreversible changes in gene expression imposed by culture in vitro. Cancer Res 2009;69:3364-73.

33. Haibe-Kains B, El-Hachem N, Birkbak NJ, Jin AC, Beck AH, Aerts HJ, et al. Inconsistency in large pharmacogenomic studies. Nature 2013;504:389-93.

34. Cancer Cell Line Encyclopedia Consortium; Genomics of Drug Sensitivity in Cancer Consortium. Pharmacogenomic agreement between two cancer cell line data sets. Nature 2015;528:84-7.

35. Gazdar AF, Gao B, Minna JD. Lung cancer cell lines: useless artifacts or invaluable tools for medical science? Lung Cancer 2010;68:309-18.

36. Kodack DP, Farago AF, Dastur A, Held MA, Dardaei L, Friboulet L, et al. Primary patient-derived cancer cells and their potential for personalized cancer patient care. Cell Rep 2017;21:3298-309.

37. Goto T. Patient-derived tumor xenograft models: toward the establishment of precision cancer medicine. J Pers Med 2020;10:64.

38. Xu C, Li X, Liu P, Li M, Luo F. Patient-derived xenograft mouse models: a high fidelity tool for individualized medicine. Oncol Lett 2019;17:3-10.

39. Ledford H. US cancer institute to overhaul tumour cell lines. Nature 2016;530:391.

40. Byrne AT, Alférez DG, Amant F, Annibali D, Arribas J, Biankin AV, et al. Interrogating open issues in cancer precision medicine with patient-derived xenografts. Nat Rev Cancer 2017;17:254-68.

41. Izumchenko E, Paz K, Ciznadija D, Sloma I, Katz A, Vasquez-Dunddel D, et al. Patient-derived xenografts effectively capture responses to oncology therapy in a heterogeneous cohort of patients with solid tumors. Ann Oncol 2017; 28:2595-605.

42. Nakajima T, Geddie W, Anayama T, Ko HM, da Cunha Santos G, Boerner S, et al. Patient-derived tumor xenograft models established from samples obtained by endobronchial ultrasound-guided transbronchial needle aspiration. Lung Cancer 2015;89:110-4.

43. Kang HN, Choi JW, Shim HS, Kim J, Kim DJ, Lee CY, et al. Establishment of a platform of non-small-cell lung cancer patient-derived xenografts with clinical and genomic annotation. Lung Cancer 2018;124:168-78.

44. Hodgkinson CL, Morrow CJ, Li Y, Metcalf RL, Rothwell DG, Trapani F, et al. Tumorigenicity and genetic profiling of circulating tumor cells in small-cell lung cancer. Nat Med 
2014;20:897-903.

45. Drapkin BJ, George J, Christensen CL, Mino-Kenudson M, Dries R, Sundaresan T, et al. Genomic and functional fidelity of small cell lung cancer patient-derived xenografts. Cancer Discov 2018;8:600-15.

46. Cho SY, Kang W, Han JY, Min S, Kang J, Lee A, et al. An integrative approach to precision cancer medicine using patient-derived xenografts. Mol Cells 2016;39:77-86.

47. Wang D, Pham NA, Tong J, Sakashita S, Allo G, Kim L, et al. Molecular heterogeneity of non-small cell lung carcinoma patient-derived xenografts closely reflect their primary tumors. Int J Cancer 2017;140:662-73.

48. Siolas D, Hannon GJ. Patient-derived tumor xenografts: transforming clinical samples into mouse models. Cancer Res 2013;73:5315-9.

49. Morton CL, Houghton PJ. Establishment of human tumor xenografts in immunodeficient mice. Nat Protoc 2007;2:247-50.

50. Shultz LD, Lyons BL, Burzenski LM, Gott B, Chen X, Chaleff S, et al. Human lymphoid and myeloid cell development in NOD/LtSz-scid IL2R gamma null mice engrafted with mobilized human hemopoietic stem cells. J Immunol 2005; 174:6477-89.

51. Ito M, Hiramatsu H, Kobayashi K, Suzue K, Kawahata M, Hioki K, et al. NOD/SCID/gamma(c)(null) mouse: an excellent recipient mouse model for engraftment of human cells. Blood 2002;100:3175-82.

52. Collins AT, Lang SH. A systematic review of the validity of patient derived xenograft (PDX) models: the implications for translational research and personalised medicine. PeerJ 2018;6:e5981.

53. Morgan KM, Riedlinger GM, Rosenfeld J, Ganesan S, Pine SR. Patient-derived xenograft models of non-small cell lung cancer and their potential utility in personalized medicine. Front On$\operatorname{col} 2017 ; 7: 2$.

54. Cuenca RE, Takita H, Bankert R. Orthotopic engraftment of human lung tumours in SCID mice for the study of metastasis. Surg Oncol 1996;5:85-91.

55. Morton JJ, Bird G, Keysar SB, Astling DP, Lyons TR, Anderson RT, et al. XactMice: humanizing mouse bone marrow enables microenvironment reconstitution in a patient-derived xenograft model of head and neck cancer. Oncogene 2016;35:290300.

56. Meraz IM, Majidi M, Meng F, Shao R, Ha MJ, Neri S, et al. An improved patient-derived xenograft humanized mouse model for evaluation of lung cancer immune responses. Cancer Immunol Res 2019;7:1267-79.

57. DeRose YS, Wang G, Lin YC, Bernard PS, Buys SS, Ebbert MT, et al. Tumor grafts derived from women with breast cancer authentically reflect tumor pathology, growth, metastasis and disease outcomes. Nat Med 2011;17:1514-20.

58. Nadkarni RR, Abed S, Draper JS. Organoids as a model system for studying human lung development and disease. Biochem Biophys Res Commun 2016;473:675-82.

59. Clevers H. Modeling development and disease with organoids. Cell 2016;165:1586-97.

60. Schutgens F, Clevers H. Human organoids: tools for understanding biology and treating diseases. Annu Rev Pathol 2020; 15:211-34.

61. Sato T, Vries RG, Snippert HJ, van de Wetering M, Barker N, Stange DE, et al. Single Lgr5 stem cells build crypt-villus structures in vitro without a mesenchymal niche. Nature 2009; 459:262-5.

62. Sato T, Stange DE, Ferrante M, Vries RG, Van Es JH, Van den Brink S, et al. Long-term expansion of epithelial organoids from human colon, adenoma, adenocarcinoma, and Barrett's epithelium. Gastroenterology 2011;141:1762-72.

63. Karthaus WR, Iaquinta PJ, Drost J, Gracanin A, van Boxtel $\mathrm{R}$, Wongvipat $\mathrm{J}$, et al. Identification of multipotent luminal progenitor cells in human prostate organoid cultures. Cell 2014;159:163-75.

64. Yin X, Farin HF, van Es JH, Clevers H, Langer R, Karp JM. Niche-independent high-purity cultures of Lgr5+ intestinal stem cells and their progeny. Nat Methods 2014;11:106-12.

65. Nikolić MZ, Rawlins EL. Lung organoids and their use to study cell-cell interaction. Curr Pathobiol Rep 2017;5:223-31.

66. He J, Zhang X, Xia X, Han M, Li F, Li C, et al. Organoid technology for tissue engineering. J Mol Cell Biol 2020;12:569-79.

67. Schwank G, Koo BK, Sasselli V, Dekkers JF, Heo I, Demircan T, et al. Functional repair of CFTR by CRISPR/Cas9 in intestinal stem cell organoids of cystic fibrosis patients. Cell Stem Cell 2013;13:653-8.

68. Li Y, Tang P, Cai S, Peng J, Hua G. Organoid based personalized medicine: from bench to bedside. Cell Regen 2020;9:21.

69. Klak M, Bryniarski T, Kowalska P, Gomolka M, Tymicki G, Kosowska K, et al. Novel strategies in artificial organ development: what is the future of medicine? Micromachines (Basel) 2020;11:646.

70. Lamers MM, Beumer J, van der Vaart J, Knoops K, Puschhof J, Breugem TI, et al. SARS-CoV-2 productively infects human gut enterocytes. Science 2020;369:50-4.

71. Creff J, Courson R, Mangeat T, Foncy J, Souleille S, Thibault C, et al. Fabrication of 3D scaffolds reproducing intestinal epithelium topography by high-resolution 3D stereolithography. Biomaterials 2019;221:119404. 
72. Homan KA, Gupta N, Kroll KT, Kolesky DB, Skylar-Scott M, Miyoshi T, et al. Flow-enhanced vascularization and maturation of kidney organoids in vitro. Nat Methods 2019;16:25562.

73. Jin B, Wu XA, Du SD. Human pluripotent stem cell-derived hepatic organoids: a promising novel model of liver diseases. Gastroenterology 2021;160:2208.

74. Tian L, Gao J, Garcia IM, Chen HJ, Castaldi A, Chen YW. Human pluripotent stem cell-derived lung organoids: potential applications in development and disease modeling. Wiley Interdiscip Rev Dev Biol 2020; 399.

75. Lambrecht BN, Hammad $H$. The airway epithelium in asthma. Nat Med 2012;18:684-92.

76. Ghosh M, Miller YE, Nakachi I, Kwon JB, Barón AE, Brantley AE, et al. Exhaustion of airway basal progenitor cells in early and established chronic obstructive pulmonary disease. Am J Respir Crit Care Med 2018;197:885-96.

77. Chambers RC, Mercer PF. Mechanisms of alveolar epithelial injury, repair, and fibrosis. Ann Am Thorac Soc 2015; 12(Suppl 1):S16-20.

78. Shanks N, Greek R, Greek J. Are animal models predictive for humans? Philos Ethics Humanit Med 2009; 4:2.

79. Benali R, Tournier JM, Chevillard M, Zahm JM, Klossek JM, Hinnrasky J, et al. Tubule formation by human surface respiratory epithelial cells cultured in a three-dimensional collagen lattice. Am J Physiol 1993;264(2 Pt 1):L183-92.

80. Rock JR, Onaitis MW, Rawlins EL, Lu Y, Clark CP, Xue Y, et al. Basal cells as stem cells of the mouse trachea and human airway epithelium. Proc Natl Acad Sci U S A 2009;106:127715.

81. Franzdóttir SR, Axelsson IT, Arason AJ, Baldursson O, Gudjonsson T, Magnusson MK. Airway branching morphogenesis in three dimensional culture. Respir Res 2010;11:162.

82. Delgado O, Kaisani AA, Spinola M, Xie XJ, Batten KG, Minna JD, et al. Multipotent capacity of immortalized human bronchial epithelial cells. PLoS One 2011;6:e22023.

83. Dye BR, Hill DR, Ferguson MA, Tsai YH, Nagy MS, Dyal R, et al. In vitro generation of human pluripotent stem cell derived lung organoids. Elife 2015;4:e05098.

84. Chen YW, Huang SX, de Carvalho AL, Ho SH, Islam MN, Volpi S, et al. A three-dimensional model of human lung development and disease from pluripotent stem cells. Nat Cell Biol 2017;19:542-9.

85. Tan Q, Choi KM, Sicard D, Tschumperlin DJ. Human airway organoid engineering as a step toward lung regeneration and disease modeling. Biomaterials 2017;113:118-32.

86. Hughes CS, Postovit LM, Lajoie GA. Matrigel: a complex protein mixture required for optimal growth of cell culture. Proteomics 2010;10:1886-90.

87. Kim M, Mun H, Sung CO, Cho EJ, Jeon HJ, Chun SM, et al. Patient-derived lung cancer organoids as in vitro cancer models for therapeutic screening. Nat Commun 2019;10:3991.

88. Shi R, Radulovich N, Ng C, Liu N, Notsuda H, Cabanero M, et al. Organoid cultures as preclinical models of non-small cell lung cancer. Clin Cancer Res 2020;26:1162-74.

89. Fujii M, Shimokawa M, Date S, Takano A, Matano M, Nanki $\mathrm{K}$, et al. A colorectal tumor organoid library demonstrates progressive loss of niche factor requirements during tumorigenesis. Cell Stem Cell 2016;18:827-38.

90. Driehuis E, Kretzschmar K, Clevers H. Establishment of patient-derived cancer organoids for drug-screening applications. Nat Protoc 2020;15:3380-409.

91. Klameth L, Rath B, Hochmaier M, Moser D, Redl M, Mungenast F, et al. Small cell lung cancer: model of circulating tumor cell tumorospheres in chemoresistance. Sci Rep 2017;7:5337.

92. Praharaj PP, Bhutia SK, Nagrath S, Bitting RL, Deep G. Circulating tumor cell-derived organoids: current challenges and promises in medical research and precision medicine. Biochim Biophys Acta Rev Cancer 2018; 1869:117-27.

93. Caponigro G, Sellers WR. Advances in the preclinical testing of cancer therapeutic hypotheses. Nat Rev Drug Discov 2011;10:179-87.

94. Pauli C, Moch H, Rubin MA. Establishment of a living biobank : improved guidance of precision cancer care with in vitro and in vivo cancer models. Pathologe 2017;38(Suppl 2):160-8.

95. Jabs J, Zickgraf FM, Park J, Wagner S, Jiang X, Jechow K, et al. Screening drug effects in patient-derived cancer cells links organoid responses to genome alterations. Mol Syst Biol 2017; 13:955

96. Takahashi N, Hoshi H, Higa A, Hiyama G, Tamura H, Ogawa $\mathrm{M}$, et al. An in vitro system for evaluating molecular targeted drugs using lung patient-derived tumor organoids. Cells 2019;8:481.

97. Wang Y, Jiang T, Qin Z, Jiang J, Wang Q, Yang S, et al. HER2 exon 20 insertions in non-small-cell lung cancer are sensitive to the irreversible pan-HER receptor tyrosine kinase inhibitor pyrotinib. Ann Oncol 2019;30:447-55.

98. Jung DJ, Shin TH, Kim M, Sung CO, Jang SJ, Jeong GS. A one-stop microfluidic-based lung cancer organoid culture platform for testing drug sensitivity. Lab Chip 2019;19:2854-65.

99. Khoo BL, Grenci G, Lim YB, Lee SC, Han J, Lim CT. Expansion of patient-derived circulating tumor cells from liquid 
biopsies using a CTC microfluidic culture device. Nat Protoc 2018;13:34-58.

100. Cattaneo CM, Dijkstra KK, Fanchi LF, Kelderman S, Kaing S, van Rooij N, et al. Tumor organoid-T-cell coculture systems. Nat Protoc 2020;15:15-39.
101. Bar-Ephraim YE, Kretzschmar K, Clevers H. Organoids in immunological research. Nat Rev Immunol 2020;20:279-93.

102. Ribas A, Wolchok JD. Cancer immunotherapy using checkpoint blockade. Science 2018;359:1350-5. 